\title{
Maximal antichains of minimum size
}

\author{
Thomas Kalinowski \\ Institute for Mathematics \\ University of Rostock, Ulmenstr. 69 \\ 18057 Rostock, Germany \\ thomas.kalinowski@uni-rostock.de \\ Uwe Leck \\ Ian T. Roberts \\ Department of Mathematics \& Computer Science \\ P.O. Box 40611 \\ University of Wisconsin-Superior \\ Casuarina, NT \\ Superior, WI 54880, U.S.A. \\ Australia 0811 \\ uleck@uwsuper.edu \\ ian.roberts@cdu.edu.au
}

Submitted: Sep 22, 2012; Accepted: Mar 27, 2013; Published: Apr 9, 2013

Mathematics Subject Classifications: 05D05,06A07,05C35

\begin{abstract}
Let $n \geqslant 4$ be a natural number, and let $K$ be a set $K \subseteq[n]:=\{1,2, \ldots, n\}$. We study the problem of finding the smallest possible size of a maximal family $\mathcal{A}$ of subsets of $[n]$ such that $\mathcal{A}$ contains only sets whose size is in $K$, and $A \nsubseteq B$ for all $\{A, B\} \subseteq \mathcal{A}$, i.e. $\mathcal{A}$ is an antichain. We present a general construction of such antichains for sets $K$ containing 2 , but not 1 . If $3 \in K$ our construction asymptotically yields the smallest possible size of such a family, up to an $o\left(n^{2}\right)$ error. We conjecture our construction to be asymptotically optimal also for $3 \notin K$, and we prove a weaker bound for the case $K=\{2,4\}$. Our asymptotic results are straightforward applications of the graph removal lemma to an equivalent reformulation of the problem in extremal graph theory, which is interesting in its own right.
\end{abstract}

Keywords: Extremal set theory, Sperner property, maximal antichains, flat antichains

\section{Introduction}

Let $n \geqslant 2$ be an integer and $[n]:=\{1,2, \ldots, n\}$. By $2^{[n]}$ we denote the family of all subsets of $[n]$ and by $\left(\begin{array}{c}{[n]} \\ k\end{array}\right)$ the family of all $k$-subsets of $[n]$. A family $\mathcal{A} \subseteq 2^{[n]}$ is an antichain if $A \nsubseteq B$ for all distinct $A, B \in \mathcal{A}$. An antichain $\mathcal{A}$ is called flat if it is contained in two 
consecutive levels of $2^{[n]}$, i.e. if $\mathcal{A} \subseteq\left(\begin{array}{c}{[n]} \\ k\end{array}\right) \cup\left(\begin{array}{c}{[n]} \\ k+1\end{array}\right)$ for some $k$. More generally, for a subset $K \subseteq[n]$, we call $\mathcal{A}$ a $K$-antichain if it contains only $k$-sets with $k \in K$, i.e.

$$
\mathcal{A}=\bigcup_{k \in K} \mathcal{A}_{k}
$$

where $\mathcal{A}_{k} \subseteq\left(\begin{array}{c}{[n]} \\ k\end{array}\right)$. The dual $\mathcal{A}^{*}$ of a family of sets $\mathcal{A}=\left\{A_{1}, \ldots, A_{m}\right\}$ is the collection $\mathcal{A}^{*}=\left\{X_{1}, \ldots, X_{n}\right\}$ of subsets of $[m]$ given by $X_{i}=\left\{j \in[m]: i \in A_{j}\right\}$ for $i \in[n]$. It is well known that a family of sets $\mathcal{A}$ is an antichain if and only if its dual $\mathcal{A}^{*}$ is a completely separating system (CSS): a CSS is a collection $\mathcal{C}$ of blocks of $[n]$ such that for each $a, b \in[n]$ there are blocks $A, B \in \mathcal{C}$ with $a \in A \backslash B$ and $b \in B \backslash A$. The dual of a flat antichain on $\left(\begin{array}{c}{[n]} \\ k\end{array}\right) \cup\left(\begin{array}{c}{[n]} \\ k-1\end{array}\right)$ is a fair CSS: a CSS in which each point occurs exactly $k$ or $k-1$ times. The consideration of minimum size CSSs led to a conjecture which subsequently became the Flat Antichain Theorem, which follows from results of Lieby [10] (see also [11]) and Kisvölcsey [9]. This theorem greatly reduced the computational search space for CSSs. It says that for every antichain $\mathcal{A}$ there is an equivalent flat antichain $\mathcal{A}^{\prime}$, where the equivalence relation is defined by: $\mathcal{A}^{\prime}$ is equivalent to $\mathcal{A}$ if and only if $\left|\mathcal{A}^{\prime}\right|=|\mathcal{A}|$ and $\sum_{A \in \mathcal{A}^{\prime}}|A|=\sum_{A \in \mathcal{A}}|A|$. Griggs et al. [6] showed that flat antichains minimize the $B L Y M$ values $\sum_{A \in \mathcal{A}}\left(\begin{array}{c}n \\ |A|\end{array}\right)^{-1}$ within their equivalence classes, and more generally, they minimize (maximize) $\sum_{A \in \mathcal{A}} w(|A|)$ for every convex (concave) function $w$. CSSs have applications in various areas including Coding Theory, Search Theory, and Topology. See [1] and [8] for applications and further references for CSSs.

A $K$-antichain $\mathcal{A}$ is called a maximal $K$-antichain if there is no $K$-antichain $\mathcal{A}^{\prime}$ with $\mathcal{A} \subsetneq \mathcal{A}^{\prime}$. For $K^{\prime} \subseteq K$, any $K^{\prime}$-antichain is also a $K$-antichain, and if it is a maximal $K$-antichain, then it is also a maximal $K^{\prime}$-antichain. The converse is not true as can be seen by the family

$$
\mathcal{A}=\{1245,2367,1389,16,17,28,29,34,35,46,47,48,49,56,57,58,59,68,69,78,79\}
$$

for $n=9$, which is a maximal $\{2,4\}$-antichain but not a maximal $\{2,3,4\}$-antichain, since $\mathcal{A} \cup\{123\}$ is an antichain properly containing $\mathcal{A}$. The collection $A$ is illustrated in Figure 1, showing a graph on 9 vertices whose edge set is the complement of $\mathcal{A}_{2}$ in $\left(\begin{array}{c}{[9]} \\ 2\end{array}\right)$, while the set of 4 -cliques is precisely $\mathcal{A}_{4}$.

We call a $K$-antichain $\mathcal{A}$ strongly maximal if it is maximal as an antichain, not just as $K$-antichain, i.e. if there is no antichain $\mathcal{A}^{\prime}$ with $\mathcal{A} \subsetneq \mathcal{A}^{\prime}$. Equivalently, $\mathcal{A}$ is a strongly maximal $K$-antichain if it is also a maximal $\{k, k+1, \ldots, l\}$-antichain, where $k$ and $l$ are the smallest and the largest element of $K$, respectively. In this paper, we always assume $1 \notin K$ and $2 \in K$, and we study the problem of determining the smallest possible cardinality of a maximal $K$-antichain.

Our approach to the minimum size of a maximal antichain is based on the graph interpretation which was used in [7] to completely resolve the problem for the case $K=$ $\{2,3\}$. To prove our asymptotic results we adapt arguments from [5] where lower bounds for the size of maximal $\{l, l+1\}$-antichains were proved. The main tool in this part is the graph removal lemma. 


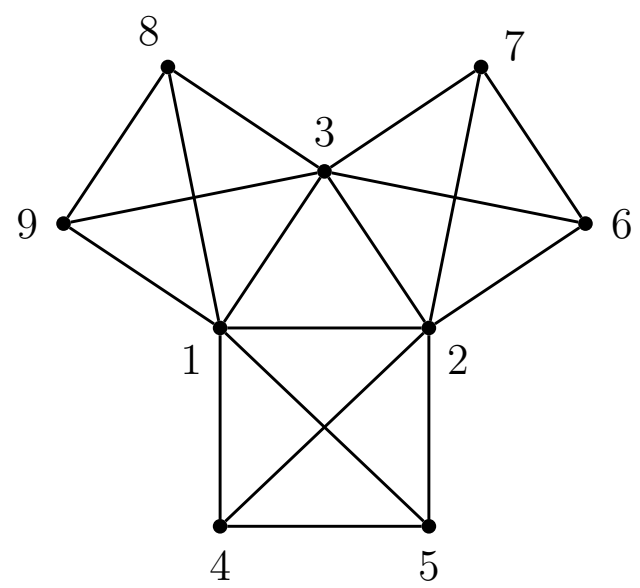

Figure 1: A graph representing a maximal $\{2,4\}$-antichain which is not maximal as a $\{2,3,4\}$-antichain, and thus also not strongly maximal.

Fact 1 (Graph removal lemma.). Let $H$ be a graph on $h$ vertices and let $\varepsilon>0$. Then there exists $\delta>0$ such that every graph $G$ on $n$ vertices containing at most $\delta n^{h}$ copies of $H$ can be made $H$-free by removing $\varepsilon n^{2}$ edges.

This was proved, using Szemerédis regularity lemma, first for $H$ being a triangle [13] and later in general [2]. See [4] for a recent proof avoiding the regularity lemma, and thus improving the bounds on $\delta$ significantly.

The paper is organized as follows. In Section 2 the problem is reformulated as a question in extremal graph theory. In Section 3 we describe a construction for graphs, which we believe to correspond to optimal antichains. Some evidence for this conjecture is provided by exhaustive search results for $K=\{2,4\}$ and small $n$ which are presented in Section 4. Section 5 contains our asymptotic results: if $3 \in K$ our construction yields the correct leading term, and for $K=\{2,4\}$ we prove a first nontrivial bound. Finally, in Section 6 we suggest some open problems for further investigations.

\section{Graph theoretical reformulation}

As proposed in [7], we associate a graph $G(\mathcal{A})=(V, E)$ with an antichain $\mathcal{A}$. The vertex set is $V=[n]$ and the edge set

$$
E=\left(\begin{array}{c}
{[n]} \\
2
\end{array}\right) \backslash \mathcal{A}_{2}
$$

We start by making some simple observations.

Lemma 2. Let $\mathcal{A}$ be a $K$-antichain on $n$ points and let $G=(V, E)$ be the associated graph.

1. Every $B \in \mathcal{A} \backslash \mathcal{A}_{2}$ induces a clique in $G$. 
2. If $\mathcal{A}$ is a maximal $K$-antichain and $A$ is the vertex set of a $k$-clique in $G$ for some $k \in K$ then there is an $A^{\prime} \in \mathcal{A}$ with $A \subseteq A^{\prime}$ or $A \supseteq A^{\prime}$. In particular, every edge of $G$ is contained in a $k$-clique for some $k \in K \backslash\{2\}$.

Proof. The first statement is just the antichain property applied to $A \in \mathcal{A}_{2}=\left(\begin{array}{c}{[n]} \\ 2\end{array}\right) \backslash E$ and $B \in \mathcal{A} \backslash \mathcal{A}_{2}$. The second statement follows from maximality, because if no such $A^{\prime}$ exists then we can add $A$ to $\mathcal{A}$ to obtain a strictly larger antichain.

The elements of the antichain are the non-edges of the graph and some cliques whose sizes are in $K \backslash\{2\}$. We say that a graph is $K$-saturated if every edge is contained in a $k$-clique for some $k \in K \backslash\{2\}$. Being $K$-saturated is a necessary and sufficient condition for a graph to be $G(\mathcal{A})$ for some maximal $K$-antichain $\mathcal{A}$. However, it is not possible to reconstruct the maximal $K$-antichain uniquely from the graph: if $\{3,4\} \subseteq K$ then a 4 -clique can (among others) come from a single 4-set or from four 3-sets in $\mathcal{A}$.

For a $K$-saturated graph $G=(V, E)$ with $V=[n]$ let $\mathfrak{A}(G)$ denote the set of all $K$-maximal antichains $\mathcal{A}$ with $G(\mathcal{A})=G$. In other words $\mathfrak{A}(G)$ is the collection of all families $\mathcal{A}=\bigcup_{k \in K} \mathcal{A}_{k} \subseteq 2^{[n]}$ such that

1. $\mathcal{A}_{2}=\left(\begin{array}{c}{[n]} \\ 2\end{array}\right) \backslash E$,

2. for all $k \in K \backslash\{2\}$ every $A \in \mathcal{A}_{k}$ induces a $k$-clique in $G$, and

3. for all $k \in K \backslash\{2\}$ and every $k$-clique $A$ in $G$, there is a $k^{\prime} \in K$ and an $A^{\prime} \in \mathcal{A}_{k^{\prime}}$ with $A \subseteq A^{\prime}$ or $A^{\prime} \subseteq A$.

A greedy choice among all the maximal $K$-antichains corresponding to a given $K$ saturated graph $G$ could be based upon the intuition to take the sets in $\mathcal{A}$ as large as possible for the given graph $G$. More precisely, for $k \in K$, let $\mathcal{C}_{k}$ denote the set of $k$ cliques in $G$ that are not contained in a $k^{\prime}$-clique for any $k^{\prime} \in K$ with $k^{\prime}>k$. Note that for $K$-saturated graphs $\mathcal{C}_{2}=\varnothing$. Let $\mathcal{M}=\bigcup_{k \in K} \mathcal{C}_{k}$ and define the maximal $K$-antichain $\mathcal{A}(G) \in \mathfrak{A}(G)$ by

$$
\mathcal{A}(G)=\left(\left(\begin{array}{c}
{[n]} \\
2
\end{array}\right) \backslash E\right) \cup \mathcal{M}
$$

However it is not necessarily the smallest maximal $K$-antichain corresponding to the graph $G$ : for $K=\{2,3,4\}$ and $G$ the complete graph on $n$ vertices we obtain $\mathcal{A}(G)=\left(\begin{array}{c}{[n]} \\ 4\end{array}\right)$, while $\left(\begin{array}{c}{[n]} \\ 3\end{array}\right)$ is a smaller maximal $K$-antichain corresponding to the same graph. But under the following sparseness condition on $G$ the $\mathcal{A}(G)$ has minimum size in $\mathfrak{A}(G)$.

Let $k^{*}$ be the minimal element of $K \backslash\{2\}$. We say that a $K$-saturated graph is $K$ sparse if for every $A \in \mathcal{M}$, there exists a $k^{*}$-clique $B \subseteq A$ such that $A$ is the unique element of $\mathcal{M}$ containing $B$. The next lemma asserts that in this situation the antichain $\mathcal{A}(G)$ has minimum size among all $K$-antichains with corresponding graph $G$.

Lemma 3. Let $G=(V, E)$ be a $K$-sparse, $K$-saturated graph, and let $\mathcal{A}=\mathcal{A}(G)$. Then $|\mathcal{A}| \leqslant\left|\mathcal{A}^{\prime}\right|$ for all maximal $K$-antichains $\mathcal{A}^{\prime} \in \mathfrak{A}(G)$. 
Proof. Let $\mathcal{A}^{\prime}$ be any maximal $K$-antichain with $G\left(\mathcal{A}^{\prime}\right)=G$. Clearly $\mathcal{A}_{2}^{\prime}=\mathcal{A}_{2}=\left(\begin{array}{c}{[n]} \\ 2\end{array}\right) \backslash E$. Let $\mathcal{C}^{*}$ be the set of $k^{*}$-cliques in $G$ that are contained in exactly one element of $\mathcal{M}$. Since $G$ is $K$-sparse there is a mapping $f: \mathcal{M} \rightarrow \mathcal{C}^{*}$ with $f(A) \subseteq A$ for every $A \in \mathcal{M}$. By the definition of $\mathcal{C}^{*}$, the mapping $f$ is injective. By the second part of Lemma 2 every element of the range of $f$ is contained in some member of $\mathcal{A}^{\prime}$. If the sets $f\left(A_{1}\right)$ and $f\left(A_{2}\right)$ are contained in the same $A \in \mathcal{A}^{\prime}$, then $A \subseteq A_{i}$ for $i \in\{1,2\}$ because $f\left(A_{i}\right)$ is contained in a unique element of $\mathcal{M}$. This, in turn, implies $A_{1}=A_{2}$ for the same reason. Hence $|\mathcal{M}|$ is a lower bound for $\left|\mathcal{A}^{\prime} \backslash \mathcal{A}_{2}^{\prime}\right|$, and this proves the statement.

The following lemma provides a characterization of the $K$-saturated graphs $G$ such that $\mathcal{A}(G)$ is a strongly maximal $K$-antichain.

Lemma 4. Let $G$ be a $K$-saturated graph for $K=\left\{2=k_{1}<k_{2}<\cdots<k_{r}\right\}$. Then $\mathcal{A}=$ $\mathcal{A}(G)$ is strongly maximal if and only if the following holds. For every $i \in\{1,2, \ldots, r-1\}$, every $q \in\left\{k_{i}+1, \ldots, k_{i+1}-1\right\}$ and every $q$-clique $B$ in $G$, we have that either

1. B contains a $k_{i}$-clique that is not contained in any $k_{i+1}$-clique, or

2. $B$ is contained in a $k_{i+1}$-clique.

Proof. Suppose $G=G(\mathcal{A})$ satisfies the condition and $\mathcal{A}$ is not strongly maximal. Then there is a set $B \notin \mathcal{A}$ that can be added such that $\mathcal{A} \cup\{B\}$ is still an antichain. The set $B$ must induce a clique in $G$ and by Lemma 2 this implies $|B| \notin K$ and $|B|<k_{r}$. Hence $k_{i}<|B|<k_{i+1}$ for some $i$. But then either of the conditions 1 (with Lemma 2) or 2 (using the definition of $\mathcal{A}(G)$ ) implies that $B$ cannot be added without violating the antichain condition. For the converse, suppose $G$ is a $K$-saturated graph containing a $q$-clique $B$, $k_{i}<q<k_{i+1}$, such that $B$ is not contained in any $k_{i+1}$-clique but every $k_{i}$-subset of $B$ is contained in a $k_{i+1}$-clique. By construction of $\mathcal{A}=\mathcal{A}(G)$, for every $k_{i}$-subset $A \subseteq B$, the antichain $\mathcal{A}$ contains a $k_{j}$-set $A^{\prime} \supset A$ for some $j>i$. But this implies that $\mathcal{A} \cup\{B\}$ is still an antichain, hence $\mathcal{A}$ is not strongly maximal.

To summarize, we have proved the following.

1. For every maximal $K$-antichain $\mathcal{A}$ there is a unique $K$-saturated graph $G(\mathcal{A})$.

2. Minimizing the size of a maximal $K$-antichain is equivalent to

$$
\max _{G}\left(|E|-\min _{\mathcal{A} \in \mathfrak{A}(G)} \sum_{k \in K \backslash\{2\}}\left|\mathcal{A}_{k}\right|\right),
$$

where the maximum is over the set of all $K$-saturated graphs $G$ and the minimum is over the set $\mathfrak{A}(G)=\{\mathcal{A}: G(\mathcal{A})=G\}$.

3. If the graph $G$ is $K$-sparse then the antichain $\mathcal{A}=\mathcal{A}(G)$ defined by (1) achieves the minimum in (2). 


\section{Constructing $K$-saturated graphs}

\subsection{A general construction}

In this section, we describe a family of $K$-saturated graphs with many edges and few cliques (corresponding to small maximal $K$-antichains). Our construction is inspired by the optimal graphs for $\{2,3\}$-antichains in [7]: We start with a complete bipartite graph with almost equal parts and add a few edges to make the graph $K$-saturated without producing too many cliques with sizes in $K$. Let $l$ be the maximal element of $K$. The vertex set is partitioned into three disjoint sets $A, B, C$ with

$$
|A|=\left\lfloor\frac{\lfloor n / 2\rfloor}{\lfloor l / 2\rfloor}\right\rfloor \cdot\lfloor l / 2\rfloor, \quad|B|=\left\lfloor\frac{\lceil n / 2\rceil}{\lceil l / 2\rceil}\right\rfloor \cdot\lceil l / 2\rceil, \quad|C|=n-|A|-|B|<l,
$$

and the edge set is determined as follows.

- The induced subgraph on $A$ is the disjoint union of $\lfloor l / 2\rfloor$-cliques.

- The induced subgraph on $B$ is the disjoint union of $\lceil l / 2\rceil$-cliques.

- Every pair $a b$ with $a \in A$ and $b \in B$ is an edge.

- The vertices in $C$ are isolated.

Clearly every clique of at least two vertices is contained in an $l$-clique, so $\mathcal{M}$ is the set of all $l$-cliques. Any $l$-clique contains vertices from $A$ and vertices from $B$, and every $k^{*}$-clique that has nonempty intersection with $A$ and with $B$ is contained in a unique $l$-clique. Thus the graph $G$ is $K$-sparse and using Lemma 3 the antichain $\mathcal{A}(G)$, defined by (1), has minimum size among the antichains in $\mathfrak{A}(G)$ and contains only 2-sets and $l$-sets. We get $|E|=(1 / 4+O(1 / n)) n^{2}$ and

$$
\left|\mathcal{C}_{l}\right|=\left\lfloor\frac{\lfloor n / 2\rfloor}{\lfloor l / 2\rfloor}\right\rfloor \cdot\left\lfloor\frac{\lceil n / 2\rceil}{\lceil l / 2\rceil}\right\rfloor=\left(\frac{1}{4\lfloor l / 2\rfloor\lceil l / 2\rceil}+O(1 / n)\right) n^{2} .
$$

Thus

$$
|E|-\left|\mathcal{C}_{l}\right|=\left(\frac{\lfloor l / 2\rfloor\lceil l / 2\rceil-1}{4\lfloor l / 2\rfloor\lceil l / 2\rceil}+o(1)\right) n^{2}= \begin{cases}\left(\frac{l^{2}-4}{4 l^{2}}+O(1 / n)\right) n^{2} & \text { for even } l \\ \left(\frac{l^{2}-5}{4 l^{2}-4}+O(1 / n)\right) n^{2} & \text { for odd } l\end{cases}
$$

The corresponding maximal $K$-antichain has size

$$
\left(\frac{\lfloor l / 2\rfloor\lceil l / 2\rceil+1}{4\lfloor l / 2\rfloor\lceil l / 2\rceil}+O(1 / n)\right) n^{2} .
$$

Note that in this construction every $q$-clique for $2 \leqslant q \leqslant l$ is contained in an $l$-clique, thus by Lemma 4 the antichains $\mathcal{A}(G)$ are strongly $K$-maximal. 


\subsection{The case $l=4$}

In the above construction we wasted the vertices in the set $C$, and the solution can be slightly improved by connecting $C$ to the rest of the graph. The best way to do this depends on the remainder of $n$ modulo $l$. Here we describe what to do for $l=4$ and $n=4 m+r(0 \leqslant r \leqslant 3)$.

$n=4 m$. In this case $A=[2 m], B=[2 m+1,4 m]$ and $C=\varnothing$ and the edge set is

$$
E=\{\{i, j\}: i \in[2 m], j \in[2 m+1,4 m]\} \cup\{\{2 i-1,2 i\}: i \in[2 m]\},
$$

and we have

$$
|E|-\left|\mathcal{C}_{4}\right|=\frac{n^{2}}{4}+\frac{n}{2}-\frac{n^{2}}{16}=\frac{3 n^{2}+8 n}{16} .
$$

$n=4 m+1$. In this case $A=[2 m], B=[2 m+1,4 m]$ and $C=\{4 m+1\}$ and the edge set is

$$
\begin{array}{r}
E=\{\{i, j\}: i \in[2 m], j \in[2 m+1,4 m+1]\} \cup\{\{2 i-1,2 i\}: i \in[2 m]\} \\
\cup\{\{4 m, 4 m+1\}\},
\end{array}
$$

and we have

$$
|E|-\left|\mathcal{C}_{4}\right|=\frac{n^{2}-1}{4}+\frac{n+1}{2}-\frac{n-1}{4} \cdot \frac{n+3}{4}=\frac{3 n^{2}+6 n+7}{16} .
$$

$n=4 m+2$. In this case $A=[2 m], B=[2 m+1,4 m+2]$ and $C=\varnothing$ and the edge set is

$$
E=\{\{i, j\}: i \in[2 m], j \in[2 m+1,4 m+2]\} \cup\{\{2 i-1,2 i\}: i \in[2 m+1]\},
$$

and we have

$$
|E|-\left|\mathcal{C}_{4}\right|=\frac{n^{2}-4}{4}+\frac{n}{2}-\frac{n-2}{4} \cdot \frac{n+2}{4}=\frac{3 n^{2}+8 n-12}{16} .
$$

$n=4 m+3$. In this case $A=[2 m], B=[2 m+1,4 m+2]$ and $C=\{4 m+3\}$ and the edge set is

$$
\begin{array}{r}
E=\{\{i, j\}: i \in[2 m] \cup\{4 m+3\}, j \in[2 m+1,4 m+2]\} \cup\{\{2 i-1,2 i\}: i \in[2 m+1]\} \\
\cup\{\{2 m, 4 m+3\}\},
\end{array}
$$

and we have

$$
|E|-\left|\mathcal{C}_{4}\right|=\frac{n^{2}-1}{4}+\frac{n+1}{2}-\frac{n+1}{4} \cdot \frac{n+1}{4}=\frac{3 n^{2}+6 n+5}{16} .
$$

The construction is illustrated in Figure 2. We conjecture that for large $n$ these graphs are best possible. 

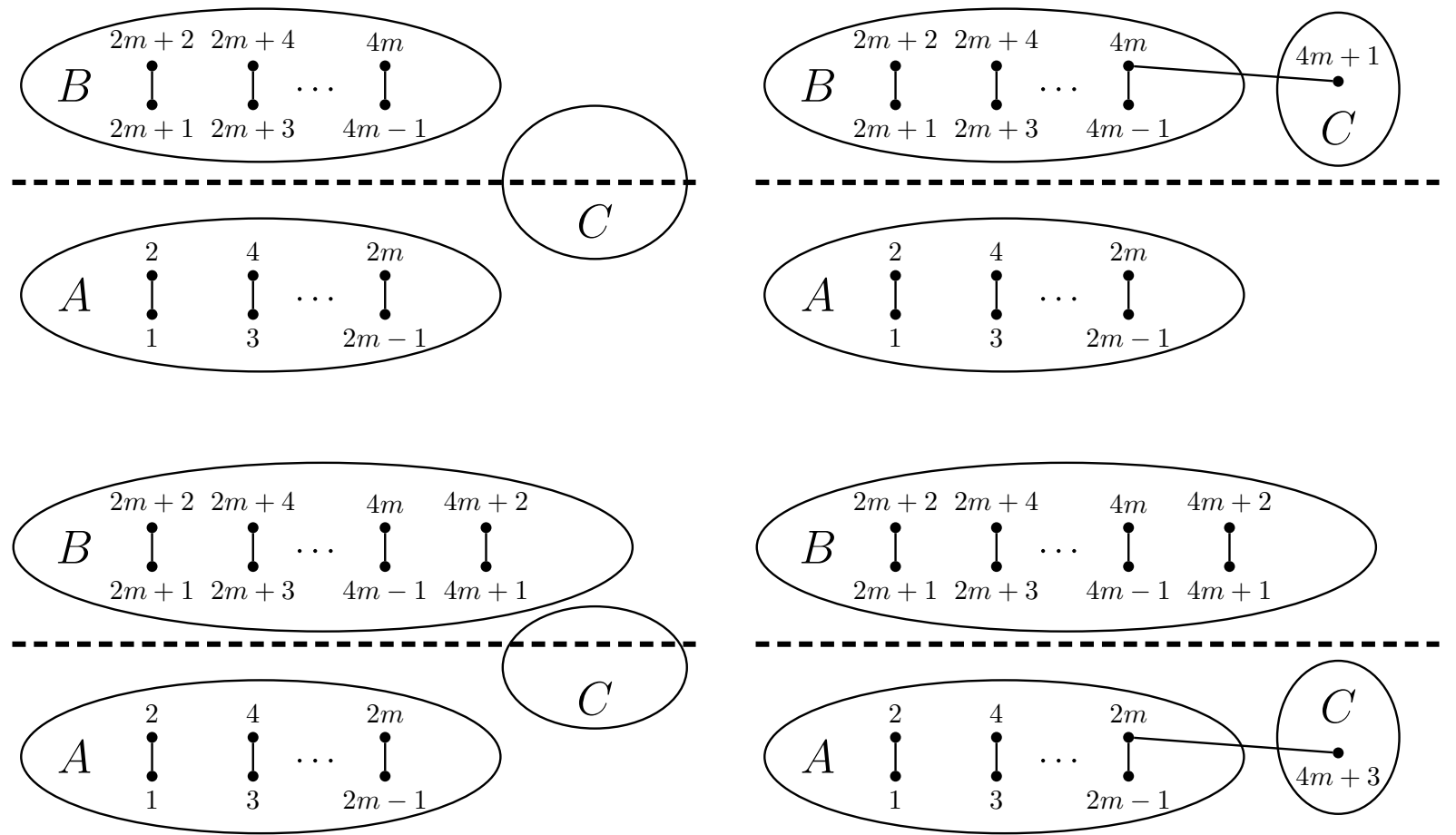

Figure 2: 4-saturated graphs for different values of $n(\bmod 4)$. All pairs of vertices on different sides of the dashed line are joined by an edge, but these edges are omitted in the pictures.

Conjecture 5. If $n$ is large, in any $\{2,4\}$-saturated graph $G$ on $n$ vertices, we have

$$
|E|-\left|\mathcal{C}_{4}\right| \leqslant f(n):= \begin{cases}\left\lfloor\left(3 n^{2}+8 n\right) / 16\right\rfloor & \text { if } n \text { is even } \\ \left\lceil\left(3 n^{2}+6 n\right) / 16\right\rceil & \text { if } n \text { is odd }\end{cases}
$$

In terms of antichains the equivalent statement is that for any maximal $\{2,4\}$-antichain $\mathcal{A}$ on $n$ points, we have

$$
|\mathcal{A}| \geqslant \begin{cases}\left\lceil\left(5 n^{2}-16 n\right) / 16\right\rceil & \text { if } n \text { is even } \\ \left\lfloor\left(5 n^{2}-14 n\right) / 16\right\rfloor & \text { if } n \text { is odd }\end{cases}
$$

\section{Small values of $n$}

In this section we present some computational results for $K \subseteq\{2,3,4\}$ and $n \leqslant 16$. These results, summarized in Table 1 , were obtained by solving binary programs for maximizing $|E|-\left|\mathcal{A}_{4}\right|$ and $|E|-\left|\mathcal{A}_{3}\right|-\left|\mathcal{A}_{4}\right|$, respectively, over all pairs $(G, \mathcal{A})$ of a $K$-saturated graph $G$ on $n$ vertices and an antichain $\mathcal{A} \in \mathfrak{A}(G)$. The binary programs were solved with the commercial MIP solver CPLEX. We make the following observations. 


\begin{tabular}{r|rr|rr|r}
\hline \hline & \multicolumn{2}{|c|}{$K=\{2,4\}$} & \multicolumn{2}{|c}{$K=\{2,3,4\}$} & \\
$n$ & $|\mathcal{A}|$ & profile & $|\mathcal{A}|$ & profile & $\left(\begin{array}{l}n \\
2\end{array}\right)-f(n)$ \\
\hline 4 & 1 & $(0,1)$ & 1 & $(0,0,1)$ & 1 \\
5 & 3 & $(1,2)$ & 3 & $(1,0,2)$ & 3 \\
6 & 6 & $(2,4)$ & 6 & $(2,0,4)$ & 6 \\
7 & 9 & $(5,4)$ & 9 & $(5,0,4)$ & 9 \\
8 & 12 & $(8,4)$ & 12 & $(8,0,4)$ & 12 \\
9 & 17 & $(11,6)$ & 17 & $(11,0,6)$ & 17 \\
10 & 20 & $(15,5)$ & 22 & $(16,0,6)$ & 22 \\
11 & 26 & $(19,7)$ & 28 & $(19,0,9)$ & 28 \\
12 & 33 & $(24,9)$ & 33 & $(24,0,9)$ & 33 \\
13 & 40 & $(29,11)$ & 41 & $(29,0,12)$ & 41 \\
14 & 47 & $(35,12)$ & 48 & $(36,0,12)$ & 48 \\
15 & 56 & $(41,15)$ & 57 & $(41,0,16)$ & 57 \\
16 & 64 & $(48,16)$ & 64 & $(48,0,16)$ & 64 \\
\hline \hline
\end{tabular}

Table 1: Computational results for small values of $n$. Columns 2 and 3 show the size of an optimal antichain $\mathcal{A}$ and its profile vector $\left(p_{k}\right)_{k \in K}$, where $p_{k}$ is the number of $k$-sets in $\mathcal{A}$. The last column contains the size of the antichain corresponding to the graphs constructed in Section 3.2.

1. For $n \leqslant 16$, the construction in Section 3.2 yields optimal $\{2,3,4\}$-saturated graphs. So the bound in Conjecture 5 might be true for $\{2,3,4\}$-graphs even without the restriction to large $n$.

2. If $n \equiv 0(\bmod 4)$, i.e. when the construction in Section 3.2 yields a regular graph, it is also optimal for $\{2,4\}$-saturation.

3. For $K=\{2,3,4\}$ all optimal examples that have been found do not contain any 3-set.

4. A particularly interesting case is $n=10$. The unique optimal $\{2,4\}$-saturated graph has the set of 4-cliques

$$
\mathcal{A}_{4}=\{1234,1567,2589,368 a, 479 a\}
$$

defining a 6-regular graph in which every vertex is contained in exactly two 4-cliques, and every edge is contained in a unique 4-clique. This can not generalize to large values of $n$, since by the removal lemma in a $\{2,4\}$-saturated graph on $n$ vertices with the property that every edge is contained in at most a constant number of 4-cliques, the number of edges is $o\left(n^{2}\right)$. 


\section{$5 \quad$ Asymptotic results}

The removal lemma argument that was used in [5] to derive asymptotic lower bounds for the size of a maximal $\{l, l+1\}$-antichain in terms of Turán numbers can be adapted to establish that for sets $K$ containing $\{2,3\}$ the construction in Section 3.1 gives the correct leading term.

Theorem 6. Let $K$ be a fixed set containing 2 and 3 , and let $l$ be its maximal element. For $K$-saturated graphs $G$ on $n$ vertices and $\mathcal{A} \in \mathfrak{A}(G)$, we have for $n \rightarrow \infty$,

$$
|E|-\sum_{k \in K \backslash\{2\}}\left|\mathcal{A}_{k}\right| \leqslant\left(\frac{\lfloor l / 2\rfloor\lceil l / 2\rceil-1}{4\lfloor l / 2\rfloor\lceil l / 2\rceil}+o(1)\right) n^{2}=\left\{\begin{array}{ll}
\left(\frac{l^{2}-4}{4 l^{2}}+o(1)\right) n^{2} & \text { for even } l, \\
\left.\frac{l^{2}-5}{4 l^{2}-4}+o(1)\right) n^{2} & \text { for odd } l .
\end{array} .\right.
$$

Proof. Let $(G, \mathcal{A})$ be a pair of a $K$-saturated graph $G=(V, E)$ and an antichain $\mathcal{A} \in \mathfrak{A}(G)$ which maximizes $|E|-\sum_{k \in K \backslash\{2\}}\left|\mathcal{A}_{k}\right|$. In particular, $\sum_{k \in K \backslash\{2\}}\left|\mathcal{A}_{k}\right| \leqslant n^{2}$, and since every triangle is contained in an element of some $\mathcal{A}_{k}$, and every $k$-clique contains $\left(\begin{array}{l}k \\ 3\end{array}\right)$ triangles, the number of triangles in $G$ is bounded by

$$
\sum_{k \in K}\left|\mathcal{A}_{k}\right|\left(\begin{array}{l}
k \\
3
\end{array}\right) \leqslant n^{2} \sum_{k \in K \backslash\{2\}}\left(\begin{array}{l}
k \\
3
\end{array}\right)=o\left(n^{3}\right) .
$$

By the removal lemma, we can make $G$ triangle-free by removing $o\left(n^{2}\right)$ edges, in other words there is a partition $E=E_{0} \cup E_{1}$ such that $\left|E_{1}\right|=o\left(n^{2}\right)$ and $E_{0}$ is triangle-free, and thus $\left|E_{0}\right| \leqslant n^{2} / 4$ by Turán's theorem. For $x y \in E_{0}$ and $k \in K$, let $\lambda_{k}(x y)$ denote the number of elements of $\mathcal{A}_{k}$ containing $x y$. Using the $K$-saturation of $G$ and observing that $E_{0}$, being triangle-free, contains at most $\lfloor k / 2\rfloor \cdot\lceil k / 2\rceil$ edges of any $k$-clique in $G$, we obtain

$$
\left|E_{0}\right| \leqslant \sum_{x y \in E_{0}} \sum_{k \in K \backslash\{2\}} \lambda_{k}(x y) \leqslant \sum_{k \in K \backslash\{2\}}\left|\mathcal{A}_{k}\right|\lfloor k / 2\rfloor \cdot\lceil k / 2\rceil \leqslant\lfloor l / 2\rfloor \cdot\lceil l / 2\rceil \cdot \sum_{k \in K \backslash\{2\}}\left|\mathcal{A}_{k}\right|,
$$

and consequently

$$
\begin{array}{r}
|E|-\sum_{k \in K \backslash\{2\}}\left|\mathcal{A}_{k}\right|=\left|E_{0}\right|+\left|E_{1}\right|-\sum_{k \in K \backslash\{2\}}\left|\mathcal{A}_{k}\right| \leqslant\left(1-\frac{1}{\lfloor l / 2\rfloor \cdot\lceil l / 2\rceil}\right)\left|E_{0}\right|+o\left(n^{2}\right) \\
\leqslant \frac{\lfloor l / 2\rfloor \cdot\lceil l / 2\rceil-1 n^{2}}{\lfloor l / 2\rfloor \cdot\lceil l / 2\rceil} \frac{o\left(n^{2}\right) .}{4}
\end{array}
$$

Corollary 7. Let $K$ be a fixed set containing 2 and 3 with maximal element $l$, and let $\mathcal{A}$ be a maximal $K$-antichain of minimum size on $n$ points. Then

$$
|\mathcal{A}|=\left(\frac{\lfloor l / 2\rfloor\lceil l / 2\rceil+1}{4\lfloor l / 2\rfloor\lceil l / 2\rceil}+o(1)\right) n^{2} .
$$


Proof. The lower bound follows from Theorem 6 and the upper bound comes from the construction in Section 3.1.

Corollary 8. Let $K$ be a fixed set containing 2 with maximal element $l$, and let $\mathcal{A}$ be a strongly maximal $K$-antichain of minimum size on $n$ points. Then

$$
|\mathcal{A}|=\left(\frac{\lfloor l / 2\rfloor\lceil l / 2\rceil+1}{4\lfloor l / 2\rfloor\lceil l / 2\rceil}+o(1)\right) n^{2} .
$$

Proof. The lower bound follows from Corollary 7 , because $\mathcal{A}$ is also a maximal $(K \cup\{3\})$ antichain, and the upper bound comes again from the construction in Section 3.1.

We expect that the statement of Theorem 6 remains true if the condition $3 \in K$ is dropped, but in this case we can prove only a weaker bound. The main difficulty comes from the fact that we are not able to deduce that an optimal graph is essentially triangle-free (meaning the number of triangles is $o\left(n^{3}\right)$ ). This weakens both bounds that are essential in the proof of Theorem 6: the upper bound for the number of edges, and the lower bound for the number of cliques in terms of the edge number. The obvious starting point for deriving bounds is that an optimal graph is almost $K_{k}$-free for every $k \in K$, which by Turán's theorem yields an asymptotic upper bound for the number of edges. Unfortunately, for $3 \notin K$ this bound is far away from the conjectured truth $n^{2} / 4$. We now consider $K=\{2,4\}$ and derive a bound for $|E|-\left|\mathcal{C}_{4}\right|$ using lower bounds for the number of triangles in graphs with more than $n^{2} / 4$ edges. Note that for sets $K$ of size 2 , the antichain $\mathcal{A}(G)$ is the only element of $\mathfrak{A}(G)$, so maximizing $|E|-\left|\mathcal{C}_{4}\right|$ is equivalent to (2). For notational convenience, in the following we write $\gamma$ for $|E| / n^{2}$. Proceeding as in the proof of Theorem 6 , we obtain a first bound.

Lemma 9. Let $G$ be a $\{2,4\}$-saturated graph on $n$ vertices. Then

$$
|E|-\left|\mathcal{C}_{4}\right| \leqslant\left(\frac{4 \gamma}{5}+o(1)\right) n^{2} .
$$

Proof. As $\left|\mathcal{C}_{4}\right|=O\left(n^{2}\right)=o\left(n^{4}\right), G$ can be made $K_{4}$-free by removing $o\left(n^{2}\right)$ edges, and we obtain a partition $E=E_{0} \cup E_{1}$ with $\left|E_{1}\right|=o\left(n^{2}\right)$ and $E_{0} K_{4}$-free, in particular any 4-clique in $G$ contains at most 5 edges from $E_{0}$. Using the 4-saturation of $G$, we obtain

$$
\left|E_{0}\right| \leqslant \sum_{x y \in E_{0}} \lambda_{4}(x y) \leqslant 5\left|\mathcal{C}_{4}\right|
$$

and finally $|E|-\left|\mathcal{C}_{4}\right| \leqslant(4 / 5)\left|E_{0}\right|+\left|E_{1}\right|=(4 \gamma / 5+o(1)) n^{2}$.

Note that this proof together with Turán's theorem already implies $|E|-\left|\mathcal{C}_{4}\right| \leqslant(4 / 15+$ $o(1)) n^{2}$ for $\{2,4\}$-saturated graphs. In order to improve the inequality, we bound the number of triangles. First we have a lower bound in terms of the number of edges. In any graph $G$ with $n$ vertices and $|E|=\gamma n^{2}$ edges, the number of triangles is at least (see $[3,12])$

$$
\frac{9 \gamma-2-2(1-3 \gamma)^{3 / 2}}{27} n^{3}
$$


On the other hand we get an upper bound for the number of triangles in terms of the number of 4-cliques.

Lemma 10. Let $G$ be a $\{2,4\}$-saturated graph on $n$ vertices. Then the number of triangles is at most

$$
\frac{(n-4)\left|\mathcal{C}_{4}\right|}{3}+o\left(n^{3}\right)
$$

Proof. Let $\mathcal{B}_{3}$ denote the set of triangles that are contained in a 4 -clique in $G$, and let $\mathcal{B}_{3}^{\prime}$ be the set of all other triangles. Note that $\left|\mathcal{B}_{3}\right| \leqslant 4\left|\mathcal{C}_{4}\right|=O\left(n^{2}\right)=o\left(n^{3}\right)$, so the number of triangles is dominated by $\left|\mathcal{B}_{3}^{\prime}\right|$. Consider four vertices $a, b, c, d$ inducing a 4 -clique in $\mathrm{G}$, and let $x \in V \backslash\{a, b, c, d\}$ be another vertex. There is at most one triangle in $\mathcal{B}_{3}^{\prime}$ of the form $x y z$ with $y, z \in\{a, b, c, d\}$. Consequently, there are at most $n-4$ triangles in $\mathcal{B}_{3}^{\prime}$ having two vertices in $\{a, b, c, d\}$, and by summation over all 4-cliques the claim follows.

Combining the two bounds for the number of triangles we obtain a second bound for $|E|-\left|\mathcal{C}_{4}\right|$ in terms of $\gamma$.

Lemma 11. Let $G$ be a $\{2,4\}$-saturated graph on $n$ vertices. Then

$$
|E|-\left|\mathcal{C}_{4}\right| \leqslant\left(\frac{2+2(1-3 \gamma)^{3 / 2}}{9}+o(1)\right) n^{2} .
$$

Now we can combine (3) and (4) to eliminate $\gamma$ and obtain an absolute bound for $|E|-\left|\mathcal{C}_{4}\right|$.

Theorem 12. Let $G$ be a $\{2,4\}$-saturated graph on $n$ vertices. Then

$$
|E|-\left|\mathcal{C}_{4}\right| \leqslant\left(\frac{2(39+\sqrt{21})}{375}+o(1)\right) n^{2}<(0.232441+o(1)) n^{2} .
$$

Proof. The right hand sides of (3) and (4) are increasing and decreasing in $\gamma$, respectively. Equating them and solving for $\gamma$ yields $\gamma=(39+\sqrt{21}) / 150$ and the result follows.

Corollary 13. Let $\mathcal{A}$ be a maximal $\{2,4\}$-antichain of minimum size on $n$ points. Then

$$
\left(\frac{219-4 \sqrt{21}}{750}+o(1)\right) n^{2} \leqslant|\mathcal{A}| \leqslant \begin{cases}\left\lceil\left(5 n^{2}-16 n\right) / 16\right\rceil & \text { if } n \text { is even } \\ \left\lfloor\left(5 n^{2}-14 n\right) / 16\right\rfloor & \text { if } n \text { is odd. }\end{cases}
$$

Proof. The lower bound is a consequence of Theorem 12, and the upper bound comes from the construction in Section 3.2. 


\section{Open problems}

An obvious next step is to improve the asymptotic bounds with the aim to replace the coefficient of $n^{2}$ in Theorem 12 by 3/16, which gives the leading term of the inequality in Conjecture 5. It seems that arguments based on the removal lemma are not sufficient to reach this goal. One reason for this failure could be that the removal lemma only requires that there are only $o\left(n^{4}\right) 4$-cliques, while in our situation we can use the stronger information that the number 4-cliques is $O\left(n^{2}\right)$. Another problem is to characterize the extremal graphs (resp. antichains) up to isomorphism. For the case $K=\{2,3\}$ this was done in [7], and it would be nice to prove that for $K \in\{\{2,4\},\{2,3,4\}\}$ and large $n$ (some slight variations of) the constructions in Section 3.2 yield all extremal graphs. In view of the computational results in Table 1 one might also try to determine if and when any maximal $K$-antichain of minimum size contains only $k$-sets and $l$-sets, where $k$ and $l$ are the smallest and the largest element of $K$, respectively.

\section{References}

[1] D. Du and F. Hwang. Combinatorial group testing and its applications, volume 3 of World Scientific Series on Applied Mathematics. World Scientific, 2000.

[2] P. Erdős, P. Frankl, and V. Rödl. The asymptotic number of graphs not containing a fixed subgraph and a problem for hypergraphs having no exponent. Graphs and Combinatorics, 2:113-121, 1986.

[3] D.C. Fisher. Lower bounds on the number of triangles in a graph. J. Graph Theory, 13:505-512, 1989.

[4] J. Fox. A new proof for the graph removal lemma. Ann. Math., 174:561-579, 2011. Preprint arXiv:1006.1300v2.

[5] D. Gerbner, B. Keszegh, N. Lemons, C. Palmer, D. Pálvölgyi, and B. Patkós. Saturating sperner families. Graphs and Combinatorics, pages 1-10, online first: doi:10.1007/s00373-012-1195-6, 2012. Preprint arXiv:1105.4453v1.

[6] J.R. Griggs, S. Hartmann, U. Leck, and I. Roberts. Squashed full flat antichains of minimum weight. Forthcoming.

[7] M. Grüttmüller, S. Hartmann, T. Kalinowski, U. Leck, and I. Roberts. Maximum flat antichains of minimum weight. Electr. J. Comb., 16:R69, 2009.

[8] M. Grüttmüller, I.T. Roberts, and L.J. Rylands. Antichains and completely separating systems - a catalogue and applications. Discrete Applied Mathematics, 2012. in press, doi:10.1016/j.dam.2012.04.016.

[9] Á. Kisvölcsey. Flattening antichains. Combinatorica, 26(1):65-82, 2006. doi:10.1007/s00493-006-0005-2

[10] P. Lieby. Extremal problems in finite sets. PhD thesis, Northern Territory University, Darwin, 1999. 
[11] P. Lieby. Antichains on three levels. Electr. J. Comb., 11:R50, 2004.

[12] A. Razborov. On the minimal density of triangles in graphs. Combinatorics, Probability and Computing, 17(4):603-618, 2008. doi:10.1017/S0963548308009085

[13] I.Z. Ruzsa and E. Szemerédi. Triple systems with no six points carrying three triangles. Combinatorics, Keszthely 1976, Colloq. Math. Soc. János Bolyai 18, 939-945, 1978. 